\title{
Singularities as Features of Deformation Grids
}

\author{
Fred L. Bookstein \\ University of Michigan, Ann Arbor, Michigan 48109 USA
}

\begin{abstract}
Biological shape differences often are represented as diffeomorphisms of a Cartesian coordinate grid. This paper suggests that their spatially discrete, localized features, for instance the details that suggest underlying developmental or pathological processes, can often be identified with variants of the singularity $(x, y) \rightarrow\left(x, x^{2} y+y^{3}\right)$. This is an unfamiliar singularity, generic of codimension 1 , at which a pair of cusps appears as a function of a parameter for "extrapolation." I introduce canonical coordinates for such singularities and show how they may be used to produce objective reports of grids encountered in an empirical context. An example is shown involving the corpus callosum in Fetal Alcohol Syndrome. These features appear to be robust under relaxation of bending energy against Euclidean distance, the analogue to multiscale analysis for discrete punctate data.
\end{abstract}

\section{Introduction}

In the rapidly growing literature of image analysis of the whole human brain, two principal methodological themes are object detection and visualization by deformation grid. The "objects" may be segmented regions (ventricle, hippocampus, tumor) from an anatomical image, or perhaps "hot spots" of metabolism exceeding baseline in a contrast of functional images. Deformations may be from atlas to patient, between different images of the same patient (pre- to intraoperative, or MR to PET), or, as will be the case in this paper, between averages of classes of patients whose contrast is important for scientific understanding of the causes and concomitants of anatomical anomalies. Yet our literature has not paid much attention to the interrelation of these two themes. Displacement and deformation grids have typically not been searched for focal features in the same way that the raw anatomical or functional images have been, and reports of features of deformation, whether focal or global, are typically not filtered through the multiscale machinery so fruitful in the contemporary methodologies of object detection for structural images and functional contrasts.

The present paper suggests a technique for bridging this gap: a method for finding focal features of deformation or displacement grids that is consistent with a multiscale approach and also with the existing biometric machinery for causes and effects of shape. Section 2 introduces a parametric template for such features, the crease singularity, and Section 3 shows how it is produced when empirical deformations are extrapolated to high multiples. Section 4 applies the method to an instructive example from the neuroanatomy of Fetal Alcohol Syndrome, an irreversible but somewhat cryptic structural-behavioral disorder, and Section 
5 shows a multiscale extension that supports a useful statistical significance test for findings like these.

\section{The basic singularity}

Consider the deformation function at right in Figure 1. (Note that the scales of the axes here are unequal.) This figure is a transformation of originally square Cartesian axes according to the composition of three functions. Two are uninteresting: the map $(x, y) \rightarrow\left(x-2 y^{2}, y\right)$, which bends lines $x=c$ into parabolas of vertical vertex tangent, and the map $(x, y) \rightarrow(x+8 y, y)$, which imposes a shear along the $x$-axis. The core of the transform, however, is quite interesting: the map $(x, y) \rightarrow\left(x, x^{2} y+y^{3}\right)$, at the left in the figure, with that curious singularity at $(0,0)$. The generic singularities of maps $R^{2} \rightarrow R^{2}$ are folds and cusps (Whitney's theorem [1]) having canonical forms $(x, y) \rightarrow\left(x, y^{2}\right)$ and $(x, y) \rightarrow\left(x, x y+y^{3}\right)$, respectively. Clearly the singularity here is neither of these. Lines $y=c$ are transformed here into a nested family of parabolas $c x^{2}+c^{3}$ whose spacing shrinks faster than curvatures as they approach the real axis. In both panels of the figure, the heavy line is the image of the original locus $x=0$. Its singularity is concealed on the left-it falls to speed zero as it traverses the axis-but, unfolded on the right, we can see how when symmetry is broken this meridian can change direction there.
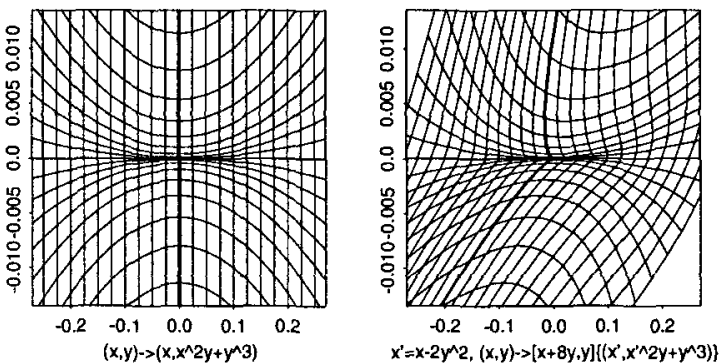

Figure 1. The basic singularity and a generalization.

Nongeneric singularities can be usefully approached as limiting cases of generic ones. In the present instance, the map $(x, y) \rightarrow\left(x, x^{2} y+y^{3}\right)$ can be viewed as the limit as $a \rightarrow 0$ of maps $(x, y) \rightarrow\left(x,\left(x^{2}-a\right) y+y^{3}\right)$, Figure 2. For $a>0$, each of these has two ordinary cusps at $\pm \sqrt{ } a$. The Jacobian of the map is negative in the lip-shaped region between the cusps. As a passes through 0, the two cusps momentarily fuse in our higher-order singularity, then vanish.

\section{How these arise in data}

The manner in which singularities like these arise as descriptors of biological shape phenomena is, needless to say, not as the limit of the parametrized map- 
pings in Figure 2. Realistic maps of one biological form onto another do not have cusps or folds. Instead, our singularity is encountered when realistic deformations are extrapolated to arbitrarily high multiples.
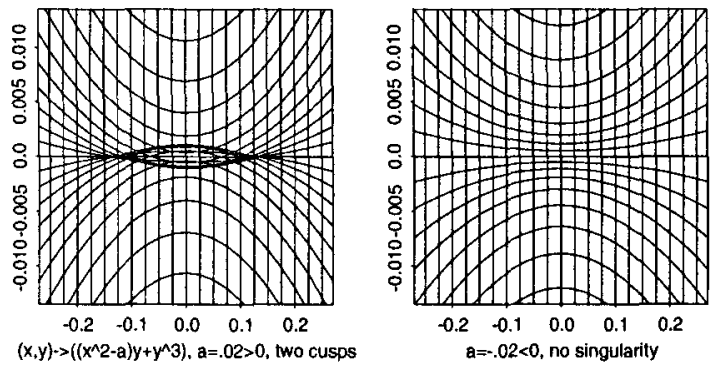

Figure 2. Limit of paired cusps: $(x, y) \rightarrow\left(\left(x^{2}-a\right) y+y^{3}, y\right)$ for $a$ near 0 .

The maps we are exploring arise as expressions of statistical contrasts corresponding to a variety of applied biometric questions, such as dimensions of greatest natural variations in shape or aspects of shape that optimally covary with exogenous causes or effects of shape. (The example here illustrates the simplest such context, the visualization of the difference between two group average shapes.) In this version of morphometrics, shape data are formalized via ordered $\mathbf{n}$-tuples of landmark points in two- or three-dimensional Cartesian space. Landmark points, such as "bridge of the nose" or "tip of the chin," have operational definitions permitting their location on specimens one by one but also are presumed to correspond on biological grounds across all the specimens of a sample. The shape of a set of landmarks is the equivalence class of their configuration under the ordinary Euclidean similarity group of translations, rotations, and changes of scale. This space is a Riemannian manifold under the celebrated Procrustes metric, submersion of the original Euclidean sum-of-squares [2]. For the purpose of this paper, we are not interested in variation of specimens within samples, but restrict our attention to representations of group average shapes, where the "average" is the form of least summed squared Procrustes distance to all the forms of the subsample. The computational geometry of the Procrustes methods has been reviewed elsewhere [3]; its details are not important for the analysis of singularities to follow.

Rather more important is the algebra of the thin-plate splines that we use for smooth interpolation between different shapes, such as averages of different subgroups of one data set. This formalism has appeared many times previously $[3,4]$. For the equations to follow we borrow the standard notations: $L$ is the bordered kernel matrix, $H$ is the vector of target landmark coordinates with three zeroes appended, $L^{-1} H$ is the vector of spline coefficients, and $L_{k}^{-1}$ is the quadratic form for bending energy.

The spline helps visualize statistical summaries based in these Procrustes coordinates in a remarkably effective manner. Any vector computed in the course of a multivariate analysis - a mean difference, for example-can be visualized 
as a deformation in this way. That the double integral of second derivatives is minimized means that the spline fits shape changes with the smallest possible variation of affine derivative (shapes of the little grid cells in these figures). If a given change of affine derivative can be managed over a larger interval, its contribution to the integral of squares is lower; hence the spline tries to represent deformations "as globally as possible." Global features of such changes are plainly visible in the grids without further parameterization. Furthermore, because the spline's coefficients $L^{-1} H$ are linear in $H$, the deformed configuration, we can unambiguously construct an $\alpha$-fold extrapolation (magnification) of the map $S_{1} \rightarrow S_{2}$ as the map $S_{1} \rightarrow S_{1}+\alpha\left(S_{2}-S_{1}\right)$.
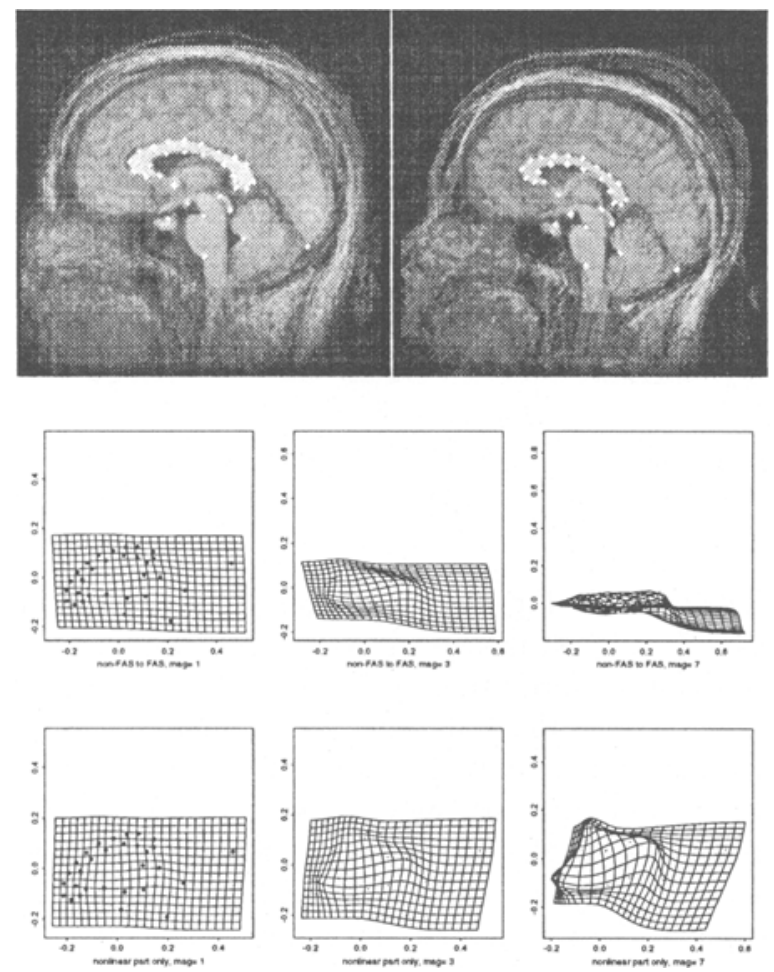

Figure 3. A two-group contrast (FAS average at top right) with ordinary (middle row) and affine-free (bottom row) extrapolates. $\alpha$, left to right: $1,3,7$.

\section{An instructive example}

Figure 3 shows, in the upper row, landmark-registered mean images from midsagittal brain MR for 4 adults with Fetal Alcohol Syndrome (FAS) and 8 persons not so affected. (Fetal Alcohol Syndrome is an irreversible process of prenatal brain damage discovered in 1973 and known to be caused by exposure to very high levels of alcohol early in fetal life.) At center left in the figure is the de- 
formation grid from left to right mean landmark configuration. In the bright white curving arch (center of the raster images, from left to top of the punctate diagrams) is the corpus callosum, the principal conduit of information between the cerebral hemispheres; cerebellum is at the lower right. The left part of the callosum is called "genu"; the bulb at the right, "splenium"; the narrowing just to its left, "isthmus."
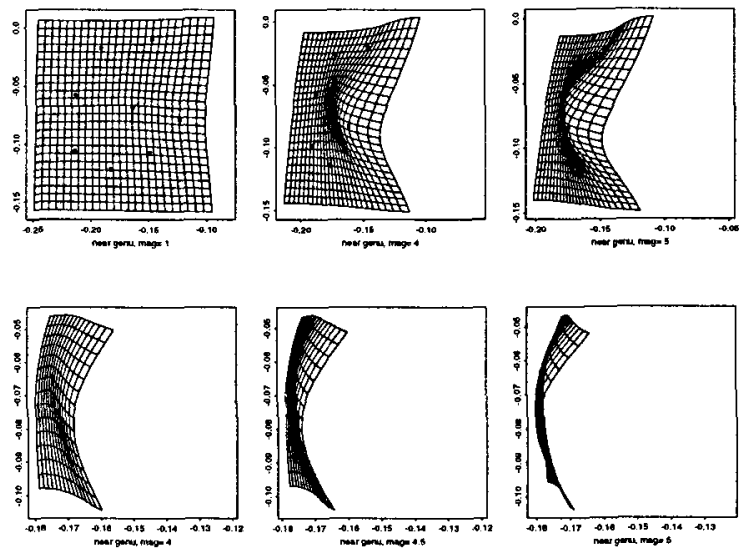

Figure 4. Zooming in on the singularity in genu, at two different scales. $\alpha$, top to bottom, left to right: $1,4,5,4,4.5,5$.

The grid at center left in Figure 3 is obviously not affine, but it is difficult to put into words exactly what it is instead. Across the middle row of the figure are extrapolations of this transformation by factors of 3 and then 7 . The bending is becoming more patent, but the collapsing of the shape at large extrapolations is interfering with our understanding. In the lower row we apply an elementary stratagem from the contemporary morphometric toolkit, removing the affine term by linear projection in the Procrustes geometry $[3,5]$. Now it is clear that there is an extended extremum of hypoplasia (compression) across the entire length of the corpus callosum. Somewhere between the threefold extrapolation and the sevenfold, the deformation goes singular in two different regions.

Figure 4 enlarges regions around genu near the extrapolation at which the singularity appears. In the top row are shown the actual deformation of the region in question and also multiples of the original transformation by four and by five; the singularity appears somewhere in between. In the lower row, a further enlargement localizes the singularity quite near the multiple of 4.5 . The higher extrapolation at lower right shows the expected paired-cusp structure.

Something here is not yet as symmetrical as it might be. In the lower left panel of Figure 4, count down about eight grid lines on the left and the right margins of the little grid cell. You will find that their images are not alignednone of the grid lines drawn appear to be transversals of the singularity. We can make this a bit clearer, Figure 5 , by rotating the data through a range of 
orientations with respect to the starting grid. At some orientations, lines slew upward along the singularity; at others, they slew downward. There is thus some orientation along which they do not slew at all, the orientation exploited at right in the figure. Here grid lines approximate a proper corner where they cross the tangent to the singularity just upstream or just downstream.
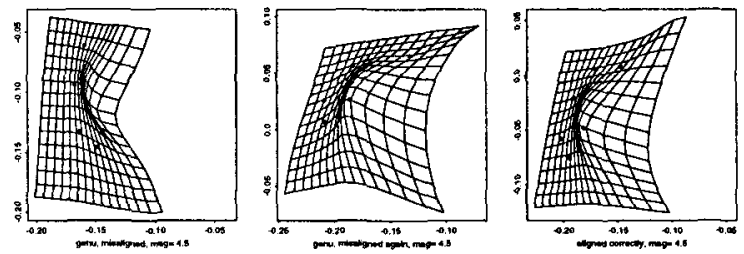

Figure 5. Rotating the starting grid through the singularity $(\alpha=4.5$ ).

What is the meaning of such a finding? I suggest that it constitutes a finite feature, a salient organizing focus for description of the map in the large. Figure 3 , lower right, shows this interpretation quite clearly. We have located the center of horizontal compression of a vertically organized dysmorphogenic field that, extended, generates the cusp visible there right up to the boundaries of the next such structure (to be encountered in Figure 6). That the singularity appears at extrapolation 4.5 means that the largest compression in any direction is by $1 / 4.5=22 \%$ of the original length, and the field of compression extends for a considerable distance upward from this feature.

These grids look remarkably like the prototype I led you to expect (recall Figure 1). The parameters of one of these features total eight: location (two), direction of the axis of the singularity, angles between the tangent to the singular locus and the principal transversal at its "corner" (two), first derivative of the map in the tangent direction, second derivative in the transversal direction, and extrapolation $\alpha$ at which all this goes singular.

This decomposition contrasts considerably with the existing methods for feature extraction from deformations. The method of biorthogonal grids, for instance [7], computes principal strains of the affine derivative at every point, but its singularities are umbilics. It offers no formalism for finding the local extrema of strain rate analogous to the analysis here. And studies that compare areas or that search for sources, sinks, or peaks (cf. [6]) have hitherto been scalar-based, lacking the very important directional features of this parameterization.

Returning to the full transformation, Figure 3 (lower right), let us track down the structure of the other salient compression, at its upper center. As Figure 6 shows, this is a composite of two singularities at about the same maximal compression $\left(\frac{1}{5}\right.$ to $\left.\frac{1}{6}\right)$ but different principal axes. In the rightmost of the two singularities, the corner of the transversal makes rather different angles with the tangent to the singularity on its two sides, as Figure 1 already prototyped (by shear along the tangent line). All along this arc of tissue (a histological locus, not a mathematical one), the average FAS case falls short of normal by about $1 / 5.5=18 \%$ in callosal thickness - that's a lot of neural tissue missing. The pair 
of findings here owe to the same alcohol-induced dysmorphogenesis that leads to the characteristic facial features by which FAS is diagnosed [8].
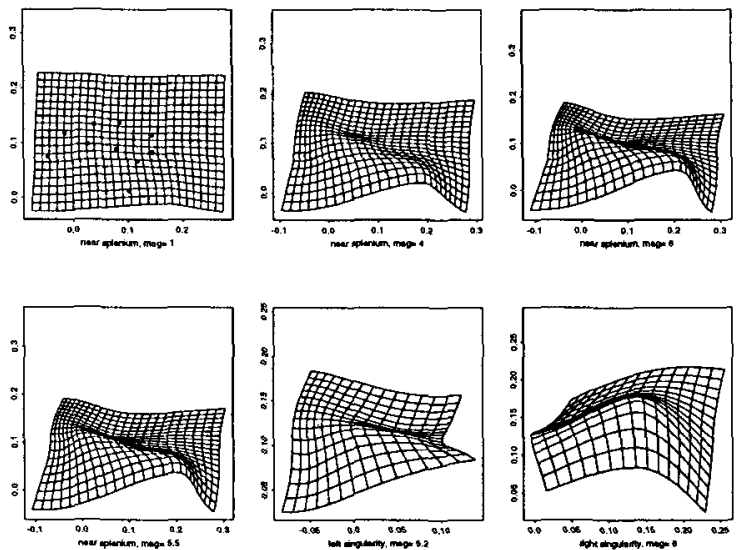

Figure 6. Near splenium, there are two more singularities, nearly collinear. $\alpha$, top to bottom, left to right: $1,4,6,5.5,5.2,6$.
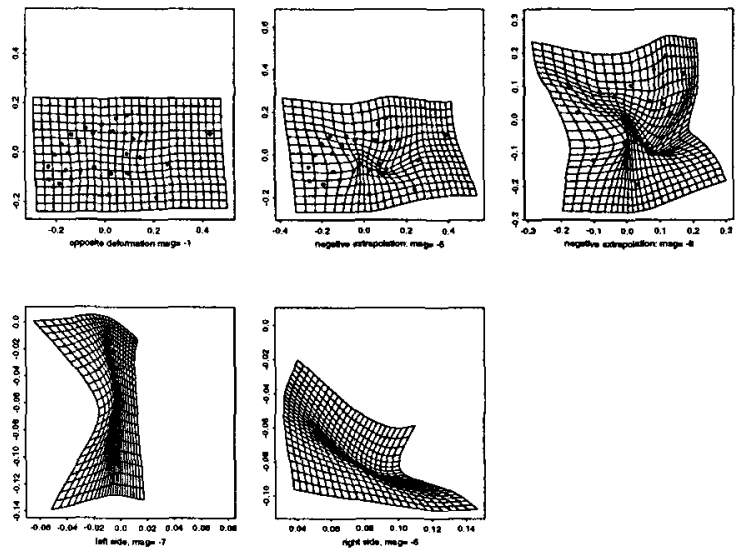

Figure 7. Negative extrapolation of the affine-free FAS grid shows two foci of expansion. $\alpha$, top to bottom, left to right: $-1,-5,-8,-7,-6$.

To complete the analysis of this transformation, we need to look at the singularities of the opposite transformation (negative extrapolations of the same map). Figure 7 shows that this transformation has two singularities of its own, rather close together, both of the same familiar structure. Because these are creases of the opposite map, they are local extrema of expansion, not compression, of the original comparison; together they account for the great whitened area at the lower right in Figure 3. These loci of greatest expansion are both within the fluid compartment of this brain section, so the expansion does the victim of the syndrome no good at all. For other empirical examples, dealing with schizophrenia, see [9]. 


\section{Relaxation preserves creases as features}

As an exact interpolant, the thin-plate spline here tracks variation in a mapping function at all pertinent scales. This is not necessarily a virtue in a world where data incorporate noise right alongside signal. By trading imprecision for bending energy, the spline can be made more similar in spirit to other energy-based methods, regularized from the outset, that compromise a pictorial mismatch energy against a complexity cost. The following approach, for instance, has been developed twice before $[10,11]$.
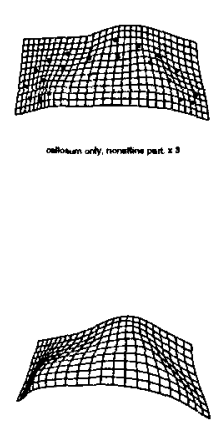

$\cos \left(\frac{10}{10}\right.$
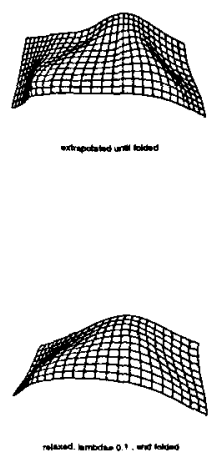
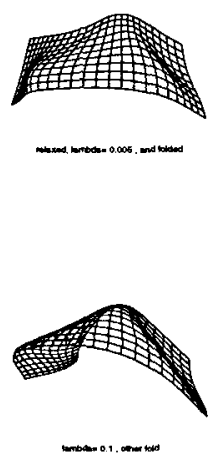

Figure 8. The relaxation that smooths the grids does not alter the creases as features. Values of $\lambda$ : upper right, .005; lower row, .02, .01, .01. The analysis is of the nonaffine signal only.

In the notation introduced earlier, reorder the Cartesian coordinates of a set of $k$ landmarks $\left(X_{i}, Y_{i}\right)$ as the $2 k$-vector $V=\left(X_{1}, X_{2}, \ldots, X_{k}, Y_{1}, \ldots, Y_{k}\right)^{t}$. To relax a perturbed configuration $V_{p}$ onto a standard configuration $V_{s}$, seek the configuration $V_{r}$ that minimizes the weighted sum of two energies. One term is the bending $\left(V_{r}-V_{s}\right)^{t} B E\left(V_{r}-V_{s}\right)$ of $V_{r}$ considered as a deformation of the standard form. (Here $B E$ is the duplication $\operatorname{diag}\left(L_{k}^{-1}, L_{k}^{-1}\right)$ of the bending-energy matrix corresponding to $V_{s}$.) The other term is "pictorial" energy, the sum-ofsquares $\left(V_{r}-V_{p}\right)^{t}\left(V_{r}-V_{p}\right)$ that corresponds to squared Procrustes distance between the relaxed landmark configuration $V_{r}$ and the data vector $V_{p}$ actually encountered. In the references cited, there was also introduced a log-likelihood $\frac{1}{2}\left(V_{r}-V_{s}\right)^{t} \Sigma^{-}\left(V_{r}-V_{s}\right)$, where $\Sigma$ is a sample variance-covariance matrix, but we shall not need that third term here.

For any relative weight $\lambda$, we seek the relaxed configuration $V_{r}$ that minimizes the weighted sum $\left(V_{r}-V_{p}\right)^{t}\left(V_{r}-V_{p}\right)+\lambda\left(V_{r}-V_{s}\right)^{t} B E\left(V_{r}-V_{s}\right)$ of the two energies. Setting the gradient of this expression to zero straightforwardly leads to

$$
V_{r} \equiv V_{r}(\lambda)=\left(I_{2 k}+\lambda B E\right)^{-1}\left(V_{p}+\lambda B E V_{s}\right) \text {. }
$$


As $\lambda$ varies, $V_{r}(\lambda)$ traverses a smooth curve in the space of landmark configurations, the charmingly named curve décolletage [10]. Note that $V_{r}(0)=V_{p}$-in the absence of any penalty for deviation from the standard $V_{s}$, the best fit to any data is the selfsame data. In the other limit $\lambda \rightarrow \infty, V_{r}$ tends to the configuration of zero bending having the least Euclidean cost: the best fit to the data by an affine transform of the standard. Because $B E$ is a function of $V_{s}$, this formulation is not symmetric in $V_{p}$ and $V_{s}$.

As $\lambda$ increases, naturally the transformation $V_{s} \rightarrow V_{r}(\lambda)$ moves closer to a uniform map. Its features, in the sense of this paper, may be restored by enhancing the extrapolation of maps $V_{s} \rightarrow V_{r}$ to compensate. (In effect we are following a curve in the two-dimensional "control space" parameterized by $\lambda$ and $\alpha(\lambda)$.) From the sequence of just-creased maps that results I have extracted the examples in Figure 8 for your consideration. Clearly these comparisons are becoming steadily smoother as the value of $\lambda$ increases; but also, just as clearly, the creases that we found in Figure 3 are fairly stable against energy-driven smoothing as operationalized by the relaxation here.

The last two frames of this figure suggest an appropriate statistical significance test for the effect claimed here. Over a range of spatial scales the deformation appears to have two creases (at two different values of $\alpha$ ) which together span nearly all of the callosal arc. Features like these are susceptible to permutationbased methods of statistical inference analogous to those now securely in place for global features [3]. Without the need for any parametric modeling, we can test the claim of a significant difference between the FAS subset and the others by examining the distribution of analogous features, at values of $\alpha$ that are no greater, when cases are permuted over group [12]. There are $\left(\begin{array}{c}12 \\ 4\end{array}\right)=495$ permuted subsettings of this data set. In addition to the one given by the actual diagnosis, 19 others show creasing along the callosum as extensive as we see here. Hence the significance level of the crease report here is $4 \%$, precisely.

\section{$6 \quad$ Future work}

The visualizations and the computational geometry associated with the search for creases are at very early stages of development. The representations here were produced slowly and interactively by hand; we need very fast algorithms for locating these structures in real data so that permutation-based statistical tests for reliability of these features can go forward in practical time. (The data set here is at the upper limit of size for which the enumeration of creased permutations is feasible by eye.) In addition to the crease singularity there are limiting cases of higher degeneracy [9] that need parametric models. In three dimensions [13] the equivalent of the catastrophe here is the emergence of a pair of the equivalently isolated singularities, swallowtails, from a diffeomorphism. We have such a finding in 3D already (see the projected images in [14]), but the parametric template for the mapping is still obscure, and the interactive search for its features is a good deal more difficult than the equivalent in two dimensions. 
Acknowledgements. The work reported here was supported in part by NIH grants DA-09009 and GM-37251 to Fred L. Bookstein. I am grateful to Hemant Tagare (Yale) for the tip about Whitney's theorem, and to my longtime collaborator Bill Green for his ewsh package permitting free play with extrapolation and grid placement in two and three dimensions. The FAS data set was gathered under NIH grant AA-10836 to Ann P. Streissguth.

\section{Literature Cited}

1. Bruce, J. W., and P. J. Giblin. Curves and Singularities, second edition. Cambridge University Press, 1992.

2. Kendall, D. G. Shape-manifolds, procrustean metrics, and complex projective spaces. Bulletin of the London Mathematical Society 16:81-121, 1984.

3. Bookstein, F. L. Shape and the information in medical images. Computer Vision and Image Understanding 66:97-118, 1997a.

4. Bookstein, F. L. Morphometric Tools for Landmark Data. New York: Cambridge University Press, 1991.

5. Bookstein, F. L. Biometrics and brain maps: the promise of the Morphometric Synthesis. Pp. 203-254 in S. Koslow and M. Huerta, eds., Neuroinformatics. Hillsdale, NJ: Lawrence Erlbaum, 1997b.

6. Thompson, P. M., and A. W. Toga. Detection, visualization, and animation of abnormal anatomic structure with a deformable probabilistic brain atlas. Medical Image Analysis 1:271-294, 1997.

7. Bookstein, F. L. The Measurement of Biological Shape and Shape Change. Lecture Notes in Biomathematics, vol. 24. Springer-Verlag, 1978.

8. Bookstein, F. L., A. P. Streissguth, P. Sampson, P. Connor, and H. Barr. Does the face predict the brain? The corpus callosum in Fetal Alcohol Syndrome. NeuroImage, submitted for publication, 1998.

9. Bookstein, F. L. Singularities and the features of deformation grids. Pp. 4655 in B. Vemuri, ed., Proc. Workshop on Biomedical Image Analysis, IEEE Computer Society, 1998.

10. Bookstein, F. L. Four metrics for image variation. Pp. 227-240 in D. Ortendahl and J. Llacer, eds., Proceedings of the XI International Conference on Information Processing in Medical Imaging. Progress in Clinical and Biological Research, vol. 363. New York: Wiley-Liss, Inc., 1990 [1991].

11. Rohr, K., H. Stiehl, R. Sprengel et al. Point-based elastic registration of medical image data using approximating thin-plate splines. Pp. 297-306 in K. Höhne and R. Kikinis, eds., Visualization in Biomedical Computing. Lecture Notes in Computer Science, vol. 1131. Springer-Verlag, 1996.

12. Good, P. Permutation Tests. Springer, 1994.

13. Whitney, H. Singularities of mappings of Euclidean spaces. In his Collected Papers, Birkhäuser, 1992, vol. 1, pp. 436-452.

14. Buckley, P. F., D. Dean, F. Bookstein et al. Three-dimensional MR-based morphometrics and ventricular dysmorphology in schizophrenia. Biological Psychiatry, in press, 1998. 